\title{
L'espace autobiographique de Barbara ou les signes en chanson de la seiche
}

\author{
Joël JULY (Aix-en-Provence)
}

Le tout est de tout dire, et je manque de mots

Et je manque de temps, et je manque d'audace

Je rêve et je dévide au hasard mes images

J'ai mal vécu, et mal appris à parler clair.

"Tout dire », Paul Eluard

(chanté par Barbara à la fête de l'Huma en septembre 1972)

\section{Summary}

More often than not, we vainly try to bring into relief the autobiographical aspects of a short poetic text. The autobiographical material being either carefully hidden or ostensibly unveiled, Philippe Lejeune coined the notion of espace autobiographique (autobiographical space), which has proved relevant to emphasize the intriguing interplay between a fiction and an attested autobiography. This methodological framework can be adjusted to the works of the French singer Barbara, and particularly to her posthumous and unfinished autobiography (Il était un piano noir, 1998) and to a selection of her songs which seem to be riddled with biographical details. Our stylistical approach aims at analysing how a feeling of ambiguity is created, intertwining false certainties and subtle indications.

\section{Introduction}

Dès 1975, Philippe Lejeune définit la notion d'espace autobiographique : "Quand ce jeu de textes comprend aussi un récit autobiographique stricto sensu, j'ai choisi de le désigner par l'expression ‘ espace autobiographique `. » (Lejeune 1996, 165) Il s'appuie sur l'exemple d'André Gide qui publie avec Si le grain ne meurt une autobiographie officielle mais très partielle malgré le protocole de sincérité au début de l'ouvrage et qui laisse donc à penser dans le même temps que c'est davantage son œuvre fictionnelle qui révèle fondamentalement la personnalité de l'écrivain. À partir de là, si aucun des romans ou des récits de Gide ne peut légitimement s'interpréter comme une autobiographie réelle, toutes les pièces qu'il crée, 
appuyées par sa correspondance, mais aussi son propre journal intime et littéraire et Si le grain ne meurt, entrent dans un espace où l'écrivain se raconte toujours, et surtout où il force le lecteur à le chercher sans cesse derrière le narrateur ou les personnages.

C'est l'analyse de Philippe Lejeune que nous voudrions appliquer à la chanteuse Barbara puisqu' elle a entretenu dans ses chansons et jusqu'à son autobiographie tardive la même illusion, engageant son public à découvrir sa vérité dans ses textes poétiques. Nous vérifierons donc tour à tour l'incomplétude et le jeu de cache-cache qu'entretiennent les deux sources autobiographiques : celle des Mémoires interrompus et celle de l'œuvre poétique chantée. Difficile de dérouler toutes les causes psychologiques et les nécessités créatives qui font préférer à un grand nombre d'artistes l'éparpillement des indices biographiques référentiels, quand un ouvrage sommatif comme une autobiographie contractuelle pourrait régler la question en une seule fois : nous l'essaierons néanmoins. Mais ce qui nous servira davantage de ligne de conduite, ce sera d'établir dans le cas de Barbara combien une poésie à vocation orale et populaire gagne en littérarité du seul fait de ces ambiguïtés.

\section{L'autobiographie proprement dite}

Célèbre auteur compositeur et interprète, Monique Serf, plus connue sous le pseudonyme de Barbara, renonce à cause de son état de santé à monter sur scène en 1994. Elle a déjà à son actif une longue carrière, entamée dès la fin des années cinquante, et un répertoire personnel de plus d'une centaine de textes. À ce moment-là de son existence, Barbara s'est très peu livrée : deux biographies sommaires ont paru, l'une rédigée par Jacques Tournier (1968) à une époque précoce, l'autre, plus tardivement, en 1986, écrite par Marie Chaix, son ancienne assistante devenue écrivaine réputée mais Barbara n'y a aucunement participé. Elle reste encore un mystère et sa vie affiche des zones d'ombre d'autant plus qu'elle a renoncé aux plateaux médiatiques et aux promotions télévisuelles depuis 1974, vingt ans auparavant. En 1994 donc, elle se retire dans sa demeure de Précy-sur-Marne, loin du strass et des paillettes de la scène. C'est pour renouer le dialogue avec le public, dit-elle, qu'elle entreprend l'écriture de ses mémoires en avril 1997, Il était un piano noir..., qui par son décès prématuré en novembre 1997 prendront le sous-titre de Mémoires interrompus (1998). Alors, l'œuvre de Barbara entre particulièrement bien dans la définition de l'espace autobiographique par Philippe Lejeune : d'un côté des textes divers n'ayant pas vocation intime mais chargés tout de même de ressemblances avec l'écrivain, les chansons, et de l'autre une autobiographie insuffisante qui rétablit quelques parcelles de vérité mais renvoie par ses vides à l'œuvre supposée fictive. "Ils ont choisi de laisser leur autobiographie incomplète, fragmentée, trouée et ouverte ", affirme Philippe Lejeune à propos de Gide et de Mauriac, « contraignant ainsi leurs lecteurs à lire dans le registre autobiographique tout le reste de leur production [...].» (Lejeune 1996, 43) ${ }^{1}$

Mais si l'acte semble délibéré de la part des deux romanciers, l'incomplétude d'Il était un piano noir..., moins volontaire, ne peut pas d'emblée apparaitre comme une stratégie de 
l'économie. D'autant que le texte, dans sa partie la plus achevée, le début, vérifie a priori tous les codes de la confession archétypique : démarrage sur la naissance et la parentèle, linéarité chronologique, pacte préliminaire de sincérité et surtout ensemble d'aveux dérangeants dans les règles du genre, un genre tauromachique, disait Michel Leiris (1998) en montrant combien l'écrivain qui utilise sa propre vie comme matériau prend des risques vis-à-vis de son entourage et de son lectorat.

Posthume et inachevée, l'autobiographie de Barbara a notamment eu le courage de révéler le traumatisme de l'enfance lié à l'inceste. Ce > détail > biographique a permis au grand public de mieux comprendre certains des mystères qui entouraient la ‘ Dame brune , il a facilité le commentaire de quelques-unes de ses œuvres les plus célèbres comme "L'aigle noir " et a suscité la mise en place du concept de ' résilience > établi par le psychanalyste Boris Cyrulnik (2001). Mais le mérite d'Il était un piano noir... ne se borne pas à ces déjà considérables atouts. Malgré son avortement, cette autobiographie déroule pour les trente premières années de la chanteuse un récit d'une grande densité où se met en place un jeu lucide, complice et tendre entre l'auteur et le lecteur. Comme Gide dans Si le grain ne meurt, l'introduction de cette œuvre, sorte de préambule, insiste sur la nécessité d'une vérité à confier aux destinataires. Elle s'adresse directement à son public devenu ses lecteurs, et justifie son entreprise. Voici l'extrait central de cette introduction :

Un soir de 1993, au Châtelet, mon cœur, trop lourd de tant d'émotion, a brusquement battu trop vite et trop fort, et, durant l'interminable espace de quelques secondes où personne, j'en suis sûre, ne s'est aperçu de rien, mon corps a refusé d'obéir à un cerveau qui, d'ailleurs, ne commandait plus rien.

J'ai gardé, rivée en moi, cette panique fulgurante pendant laquelle je suis restée figée, affolée, perdue.

J'ai dû interrompre le spectacle pendant quelque temps, puis définitivement.

Je suis quand même partie en tournée, deux mois après ; je raconterai ce que fut cette tournée, du premier jour au dernier soir.

Ensuite j’ai regagné Précy avec un manque immense, et, durant deux ans, j'ai fait le deuil d'une partie de ma vie qui venait brusquement de se terminer.

Écrire, aujourd'hui, est un moyen de continuer le dialogue.

Pourquoi ai-je accepté, pour la première fois, de parler d'un avant ? Parce que je suis la seule à pouvoir le faire ! Je vais donc essayer, même si le temps déforme les images qui deviennent floues ou, au contraire, trop précises, trop joyeusement ou douloureusement exactes.

J'ai beaucoup de travail qui m'attend, mais c'est un travail que j'aime, je ne vais pas m'en plaindre. (Barbara 1998, 9-10)

Ce texte est une introduction qui se place en épigraphe de l'autobiographie proprement dite. Il fonctionne comme une lettre dont le lecteur est le destinataire, comme un commentaire de la démarche de vérité qui va suivre et de l'entreprise (Barbara parle de 〈 travail 〉) qui va 
s'accomplir. La question rhétorique (« Pourquoi ai-je accepté, pour la première fois, de parler d'un avant ?") sert à introduire des explications : Barbara est consciente d'avoir volontairement jusqu'alors refusé de parler d'elle et de s'être cachée derrière son œuvre chantée. Les précisions temporelles, qui abondent en aval et en amont de cet extrait, montrent qu'elle est déjà pénétrée du souci de situer les événements de sa vie. De même, sont déjà au service de l'autobiographie toutes les indications liées à son sentiment. Le texte qui se veut encore une déclaration d'amour associe la démarche rétrospective comme " un moyen de continuer le dialogue ". De fait, elle n'affiche pas, contrairement à d'autres autobiographes comme JeanJacques Rousseau, sa sincérité : pour elle, c'est une évidence puisqu'il s'agit d'un " dialogue " et non d'une confession. Surtout, Barbara est consciente d'être bien trop célèbre pour ne pas attirer les biographes et les rumeurs (auxquelles elle dit " refermer [sa] porte " dans la chanson "L'enfant laboureur » en 1973 ; cf. Barbara 2012, 179) mais elle est seule à pouvoir dire la vérité, en toute simplicité. La transparence est obligatoirement induite de cette postulation : sa vie serait déformée si elle laissait le soin aux autres de l'écrire.

Mais cette volonté de transparence est également induite par le mouvement du texte et la rupture qu’il fige : "Plus jamais le rideau qui s'ouvre, plus jamais le pied posé dans la lumière sur la note de cymbale éclatée. / Plus jamais descendre vers vous, venir à vous pour enfin nous retrouver. " (Barbara 1998, 9)

Tout le début de ce texte liminaire met en anaphore la double négation "plus jamais » et énumère en un parallélisme flamboyant les préparations de la chanteuse avant un récital. Ce renoncement douloureux qui s'apparente à un deuil donne à toute l'autobiographie tardive une légitimité. Loin d'un geste commercial ou stratégique, Barbara prend la plume par nécessité. Si elle dit s'être déjà confiée à son public à travers ses chansons, leur séparation irréversible depuis 1994 donne une urgence particulière à son récit de vie. ${ }^{2}$ Alors que Gide étudie soigneusement la date à laquelle il pourra (enfin) publier Si le grain ne meurt, alors qu'il sait qu'il reste encore des ouvrages à venir, Barbara se retrouve en avril 1997 dans une impasse de création artistique qui fait douleur. En insistant dès le début du texte sur cette plaie, elle établit une espèce de contrat supplémentaire : non seulement elle accepte de parler d'un avant dans un récit rétrospectif mais elle sous-entend qu'il sera définitif et testamentaire.

D’ailleurs, la ‘ Dame de Précy > qui prend la plume et se décrit au milieu de son jardin à la fin de cette introduction n'est déjà plus tout à fait dans les mêmes dispositions que la chanteuse. La première image qu'offre Barbara est celle, très traditionnelle, de l'artiste de variétés, et notamment de la femme de scène, embellie et transformée par le maquillage ; c'est celle que son public connait, celle qu'elle lui a laissée :

Plus jamais ces heures passées dans la loge à souligner l'œil et à dessiner les lèvres avec toute cette scintillance de poudre et de lumière, en s'obligeant avec le pinceau à la lenteur, la lenteur de se faire belle pour vous.

Plus jamais revêtir le strass, le pailleté de velours noir.

Plus jamais cette attente dans les coulisses, le cœur à se rompre. (Barbara 1998, 9) 
En choisissant l'angle de vue de sa préparation pour la scène, Barbara prend l'apparence d'une amante qui se prépare à un rendez-vous amoureux. Toute cette cérémonie insiste sur des aspects sensuels : " œil ", « lèvres ", " cœur à se rompre », "se faire belle ", mais accentue en même temps les aspects artificiels ou factices dont pouvait se parer son ancienne personnalité. Barbara qui se souvient de la manière dont elle se transfigurait pour son public procède à rebours à une sorte de blanchiment. Elle semble ôter le noir qui lui colle à la peau : la deuxième partie du préambule montre, par opposition, une Barbara mise à nu, qui affiche son âge sans coquetterie. "Il est six heures du matin, j'ai soixante-sept ans, j'adore ma maison, je vais bien. De la pièce où j'écris, je vois le jardin : les premières roses sont apparues et la glycine blanche dégouline dans le patio. » (Barbara 1998, 11)

Barbara, habituée à se grimer pour nous séduire, habituée aux mystères pour nous subjuguer, prend délibérément le parti de la vérité et ce préambule sert d'enseigne à une démarche autobiographique en pleine lumière.

\section{Tout dire... ou dire l'essentiel ?}

Pourtant, même dans les premiers chapitres entièrement rédigés, Barbara comme Gide n'a pas l'ambition de tout dire. Elle a certes renoué des contacts anciens (notamment son époux belge Claude Sluys) pour étayer sa mémoire mais le récit linéaire s’interrompt parfois, dérangé par le présent de l'écriture. Par exemple, Barbara crée la surprise en sortant de la chronologie autobiographique pour revenir au présent d'énonciation. Ce sont simplement des oiseaux qui l'ont distraite. Elle ne peut s'empêcher de le noter et de rendre hommage à leur énergie : "Les oiseaux piaillent au jardin : des verdiers, des mésanges à tête noire, le ‘ pleu-pleu > (c'est un minuscule oiseau qui annonce la pluie). Cela vous fait parfois un vacarme assourdissant les oiseaux... » (Barbara 1998, 34)

Ne nous leurrons pas. Barbara sait ce qu'elle fait. Son interruption est très volontaire : le chant, la tête noire, la pluie qui la ravit (au double sens du terme) à l'égal de l'automne, sont autant de signes qui peuvent faire sens. En décrivant des oiseaux, elle continue de parler d'elle. En feignant d'être dérangée par eux, elle montre implicitement la fatigue de la ‘ Dame de Précy s. Mais l'attitude dilettante de cette autobiographe occasionnelle cadre mal avec le sérieux de la démarche et l'enjeu de la confession. De fait, les textes épars, intitulés Fragments qui s'inscrivent dans la deuxième partie de sa vie ${ }^{3}$ évoquent peu d'anecdotes précises ou de révélations intimes : quelques souvenirs de récital, beaucoup de textes qui rendent hommage à des personnalités célèbres qu'elle a côtoyées, quelques allusions tout de même très révélatrices à son sentiment de justice et à ses colères. Mais tout se passe comme si ce qu'elle avait à dire de si urgent avait déjà été dit dès les premières pages. Bien sûr, en révélant dans Il était un piano noir... la relation incestueuse que son père lui a fait subir, entre dix ans et demi et seize ans, Barbara transforme son récit de vie en aveu et offre une parenté précieuse avec André Gide : 
J'ai de plus en plus peur de mon père. Il le sent. Il le sait. J'ai tellement besoin de ma mère, mais comment faire pour lui parler ? Et que lui dire ? Que je trouve le comportement de mon père bizarre? Je me tais.

Un soir, à Tarbes, mon univers bascule dans l'horreur. J'ai dix ans et demi.

Les enfants se taisent parce qu'on refuse de les croire.

Parce qu'on les soupçonne d'affabuler.

Parce qu'ils ont peur.

Parce qu'ils croient qu'ils sont les seuls au monde avec leur terrible secret.

De ces humiliations de l'enfance, de ces hautes turbulences, de ces descentes au fond du fond, j'ai toujours resurgi [...] pour me sentir un jour purifiée de tout, longtemps après. (Barbara 1998, 31)

L'inceste sera encore rappelé lorsque Barbara évoque les souvenirs de l'été 1946, elle a déjà seize ans. Elle fugue pour prévenir la gendarmerie. Son père la récupère au poste, l'affaire est enterrée mais cet acte de dissuasion semble enfin la libérer des maltraitances paternelles. Quelque temps plus tard, en 1949, le père disparaît sans donner de nouvelles. Barbara ne le reverra que mort (ou peut-être lors d'éventuels croisements rue de Vitruve ou en plein Paris ; cf. Lehoux 2007, 49-50), et la chanson "Nantes ", pourtant fantasmée, insiste sur cette réalité cruelle des retrouvailles ratées.

Comme Si le grain ne meurt dont Philippe Lejeune pense qu'il n'a été écrit que pour révéler l'homosexualité et l'onanisme de l'écrivain Gide, Il était un piano noir... se libère rapidement d'un fardeau longtemps retenu et finalement tout le projet autobiographique de Barbara se concentre dans cette révélation.

En fait l'autobiographie de Gide n'a qu'un rôle latéral dans sa construction autobiographique : loin d'être un tout, ce n'est qu'un biais qui s'ajoute à d'autres biais. Biais privilégié peut-être en ce qu'il révèle l'existence de l'espace autobiographique; mais néanmoins limité [...]. Si Gide a choisi d'écrire un récit autobiographique, alors que cela était si contraire à son projet, c'est avant tout parce que c'était la seule manière de mettre fin au mensonge et à l'hypocrisie sur un point particulier : sa vie sexuelle. Ce qu'il apprécie dans le pacte autobiographique, c'est sa valeur d'engagement. L'écriture et la publication de l'autobiographie avaient à ses yeux valeur d'acte autant que d'œuvre. Il s'agissait de rompre l'écran des fictions et de dire la vérité, comme un aveu ou comme un défi [...]. Pour éclairer ce biais particulier, un récit rétrospectif clairement assumé était irremplaçable. (Lejeune 1996, 173)

Dans un même état d'esprit, Barbara a longtemps masqué dans ses chansons la réalité de l'inceste. Par exemple, il est à présent assez courant de voir dans la chanson "Au cœur de la nuit ", qu'elle a interprétée de 1967 à 1968, puis qui a, par la suite, été évincée de ses récitals et de ses disques, une réminiscence des assauts paternels de l'enfance. Or dans la deuxième biographie consacrée à Barbara en 1986, Marie Chaix, qui fut entre 1966 et 1968 la 
secrétaire particulière de la chanteuse en même temps qu'une complice fidèle, s'interrogeait déjà, sans pressentir explicitement l'inceste, sur ces visites nocturnes, ces rencontres funestes, ces balades somnambuliques que la chanson évoque :

Moi qui ne me souviens jamais

D’un passé qui m'importune

C'est drôle j'ai gardé le secret [...]

D'une nuit de mon enfance. [...]

Il y eut un bruissement d'ailes, [...]

Un bruit sourd venant d'outre-tombe, [...]

Qui es-tu pour me revenir

Quel est donc le mal qui t'enchaîne [...]

Si tu le veux j'irai encore $[. .$.

Pour qu'enfin tu puisses dormir [...]

Que tu finisses de mourir [...] («Au cœur de la nuit »)

Marie Chaix fait avec une grande perspicacité le parallèle entre «Au cœur de la nuit » qui précède et « Nantes », chanson de 1963 dédiée explicitement ${ }^{4}$ à son père, mais aussi "L'aigle noir " :

Le voyage au creux de la nuit s'arrête là. Nous ne saurons pas quelle ombre se faufilait entre les branches. Puis revient - comme dans "Nantes » - la petite ritournelle du début de la chanson [...]. A-t-elle erré longtemps "à demi somnolente " avant de s'endormir, belle au bois, auprès d'un lac ? Est-ce la même que l'on retrouve... quatre ans plus tard, quand soudain, "surgissant du passé, surgit un aigle noir [...] » ? L'aigle noir regagne son ciel sans elle, mais il est l'image de sa délivrance. Le vagabond de "Nantes » a enfin trouvé le repos dans son cœur, elle aura le droit de préférer vivre et de quitter ce deuil-là. (Chaix 1986, 69-70)

De fait, "L'aigle noir » qui date de 1970, reprend un vers de la chanson " Nantes », comme pour marquer leur parenté : "Il m’était revenu » (vers 23 de " L'aigle noir »).

Il était donc très tentant comme Marie Chaix, dès 1986, de reconnaître l'image du père dans cet aigle sombre et royal qui vient hanter un rêve cauchemardesque mais Barbara ne fit aucun commentaire à l'époque de la publication de cette biographie. ${ }^{5}$ Cette importance d'" Au cœur de la nuit ", la moins connue des trois chansons du triptyque paternel, sera finalement mise en évidence par Barbara elle-même : en 1993, pour son dernier Châtelet, Barbara envisage de reprendre ce titre ancien comme le prouve une première épreuve de la play-list. ${ }^{6}$ Au dernier moment, l'ordre des chansons est remanié et Barbara supprime la reprise d' "Au cour de la nuit ». Elle ne se sent pas encore assez mûre pour l'exhumer. Or le dernier acte de la chanteuse de son vivant fut le choix de quarante de ses chansons pour une compilation chez Philips qui sortit quelques jours avant sa mort, en novembre 1997. «Au 
coeur de la nuit » est le huitième titre qu'elle retint, trente ans après sa création, trente ans après son éviction. Il est évident que ce choix fut concomitant de l'ébauche de ses mémoires. Barbara a elle-même compris la nécessité de mettre au jour ce triptyque. Elle a surtout accepté de reprendre celle des trois chansons qui était la plus révélatrice de ce " passé qui [1]'importun[ait] » («Au cœur de la nuit »). S’il fallait un dernier argument pour prouver le lien qui unit ces trois pièces, nous évoquerions l'existence d'images communes entre «Au cour de la nuit » et " L'aigle noir » :

Surgissant de l'allée obscure

Il y eut un bruissement d'ailes (vers 30-31, « Au cœur de la nuit ») ;

Près de moi dans un bruissement d'ailes [...]

Surgissant du passé (vers 8 et 19, «L'aigle noir»).

En plus de la nuit et du silence, le surgissement et le bruissement sont deux motifs récurrents, comme la formule " Il m’était revenu » liait " Nantes " à " L'aigle noir ».

D'après les commentateurs (Garcin 1999, 27 et 59), tout un pan de l'œuvre noire de Barbara vient confirmer l'impossible deuil du père. Pour n'être pas parvenue à temps dans "Nantes », la ' Chanteuse de minuit > a gardé ses plaies béantes et s'est en quelque sorte condamnée à un envoûtement : l'image paternelle, toujours dangereuse et nauséeuse, a continué de la harceler. Elle donne naissance à trois chansons, formant triptyque, qui rencontrèrent des succès variés. Pourtant, ce père criminel et banni qu'elle nous présente sous son vrai jour dans ses confessions posthumes, Barbara ne l'a pas désiré ainsi dans "Nantes ", et les variantes multiples de la chanson donnent parfois de lui une image très élogieuse, de même qu'elle a pris soin d'en camoufler l'identité dans les deux autres chansons. L'autobiographie tardive intervient en quelque sorte pour mettre les pendules à l'heure et, comme Gide, Barbara choisit à ce moment-là la forme officielle du récit autobiographique assumé. Ce n'est qu'en état de manque (son public), poussée par une fin de vie et consciente qu'il lui faut raconter deux ou trois choses qu'elle sait d'elle que Barbara consent à imprimer son pseudonyme sur la couverture d'un récit à visée totalisatrice.

Car, en plus de l'aveu pudique et allusif d'un inceste subi qui occupe seulement la page 30, deux épisodes marquants apparaissent plus longuement, révélations cruciales qui rendaient cette autobiographie nécessaire. Ce sont d'abord l'épisode de la déchéance bruxelloise (la tentation de la prostitution relatée de la page 74 à la page 80 ), puis la réalité de l'épisode nantais (comment elle fut prévenue de la mort de son père, de la page 126 à la page 137). Mais là encore les textes sont très narratifs : Barbara restitue la tonalité du drame en évoquant avec précision les émotions mais elle laisse l'explication ouverte et n'élabore aucune interprétation. Le reste de la partie rédigée d'Il était un piano noir..., non qu'il ne soit intéressant pour le biographe ou le discographe, cherche moins à expliquer la personnalité de l'auteur qu'à retrouver les dates et les personnes qui ont constitué son existence. La plupart du temps, le lecteur est confronté à de considérables raccourcis : 
Du retour en octobre 1945 : rien.

Je ne me souviens de rien. (Barbara 1998, 45)

Le soir, lorsque je pars chanter, je laisse allumée une lampe de chevet en osier pour rendre plus chaleureux mes retours en pleine nuit...

Court-circuit.

Incendie.

Tout se consume. Les braises incandescentes s'arrêtent juste aux pieds du piano.

Pompiers. Eau. Cendres. Plus de lit. (Barbara 1998, 112)

En janvier 1958, réengagement à L'Écluse renouvelé de mois en mois, puis d'année en année, pendant six ans! (Barbara 1998, 113)

De telles faiblesses dans le plaisir de narrer pourraient faire douter du vœu liminaire de la chanteuse transformée en autobiographe. Certes, le stylisticien trouve son compte dans ces formules lapidaires, presque insouciantes, à travers lesquelles il entend la plume caractéristique des dernières chansons en vers libres et heurtés de Barbara. Mais dans leur aridité confidentielle, elles dévoilent justement, comme chez Gide qui préfere biaiser, que Barbara, lorsqu'elle n'avance plus masquée, ne prend plus de plaisir et se sent mal à l'aise en pleine lumière. Ce n'est donc pas seulement le décès de la chanteuse qui fait de ses mémoires une autobiographie inachevée ; d'emblée, Barbara avait opté pour une écriture partielle qui se contenterait de révéler, sous son propre nom, les quelques étapes de sa vie qui ne pouvaient plus être tues. La personnalité, elle était déjà construite dans et par les chansons qu'elle avait écrites : il n'était nul besoin de souligner les traits au risque de les déformer. Philippe Lejeune explique ainsi le comportement de Gide et pour Barbara je le suivrai volontiers en canon : " [I]l a choisi ces limites, et il en a savamment joué pour ne faire de son autobiographie qu'un texte particulier, où l'aveu passe, sans que l'histoire s'y fixe. » (Lejeune 1996, 179)

Malgré la tentation d'une autobiographie complète et la promesse d'un travail méticuleux, il semble que Barbara ait continué à souffrir du complexe de la seiche. C'est dans la page qui précède, dans Il était un piano noir..., l'aveu des harcèlements paternels, que Barbara fait un parallèle avec cet animal a priori repoussant :

La seiche est une espèce de poisson-mollusque qui, pour se protéger, diffuse une encre noire qui la dissimule au pêcheur et à ses autres prédateurs. Lorsque je ne voulais plus voir ni entendre, j'appuyais dans ma tête sur une gâchette secrète et me retrouvais immédiatement coupée du monde, les genoux repliés sous le menton, devenue inatteignable, à l'abri de herses et de murailles invisibles. Je pouvais rester ainsi une journée entière sans qu'on réussisse à me faire bouger. (Barbara 1998, 30-31)

Placé à cet endroit de la chronologie, on comprend que ce souvenir psychologique découle à la fois des traumatismes liés au viol mais également incarne une des méthodes spontanées 
de l'enfant pour les évacuer. La seiche qui se couvre de noir, c'est l'enfant qui se tait et qui trouve une échappatoire dans un suicide fictif ou peut-être seulement un univers fictif dans lequel l'inceste n'aurait pas eu lieu. Mais cet animal sombre qui protège ses sentiments et limite ses réactions a certainement partie liée avec la résilience de Barbara. De fait la chanteuse n'hésite pas à s'entourer dans ses chansons d'un bestiaire nocturne : les papillons de nuit dans "Fragson " et " John Parker Lee ", les souris dans "Gueule de nuit " ou " Les insomnies ", les loups dans "Nantes ", "Ce matin-là ", "Parce que ", " Mes hommes » et "Cet assassin » et l'aigle noir aux « plumes couleur de la nuit » ("L'aigle noir »). Le noir de Barbara, son hommage à la lune et ces animaux sombres sont paradoxalement le reflet d'une attitude de défense quand certains n'ont voulu y voir que du maniérisme ou du mysticisme. Barbara s'exhibe mais en noir. Toute l'œuvre entretient donc ce rapport complexe d'une personnalité opaque et chargée de mystère qui certifie par ailleurs qu'elle n'invente rien. L'espace autobiographique de Barbara prend donc naissance dans ses premiers textes où elle est déjà la seiche, la ‘ Chanteuse de minuit `, la ‘ Dame brune `, la louve ${ }^{7}$, celle qui dit je continuellement, jusqu'à une autobiographie terminale qui avoue l'innommable sans révéler l'essentiel.

\section{Et tout l'espace autour... : poétique de la seiche}

Pourtant, à la différence de Gide dont l'espace autobiographique est constitué de textes divers mais la plupart du temps narratifs, Barbara n'a jamais écrit que des textes courts et particulièrement lyriques. Peut-il exister des poèmes autobiographiques et surtout peut-il exister des poèmes plus autobiographiques que les autres? Peut-on dire qu'une écriture poétique puisse sortir du lyrisme commun pour se couvrir d'une dimension réellement autobiographique et si oui : quels critères retenir ? Avant d'envisager à propos de Michel Leiris les mots ‘ autobiographie > et ‘ poésie `, Philippe Lejeune ménage lui-même cette sage restriction : "Confronter deux mots aux contours aussi incertains, c'est s'exposer à soulever des problèmes vagues et immenses, et peut-être de faux problèmes. » (Lejeune 1996, 245)

Essayons d'y voir clair. La chanson invite souvent l'auditeur à identifier spontanément le canteur, c'est-à-dire l'instance qui dit je en chanson, et l'interprète ; c'est encore plus vrai si l'interprète est également le parolier, et lorsque l'auditeur devient le spectateur de cet interprète chantant son propre texte. Le contact auditif avec la voix et la vision du personnage de scène suscitent forcément une émotion qui donne l'illusion d'une proximité entre le chanteur et les mots qu'il prononce (cf. July 2016). Une voix et un corps les assument; de plus, le public demande souvent des comptes à l'artiste pour les idées qui apparaissent dans ses chansons, comme si, dans cet art populaire qu'est la chanson, l'art cessait d'être perçu comme une médiation pour n'être plus que le vecteur de la pure spontanéité. Pourtant, on peut aussi penser avec Paul Zumthor que l'auditeur, sorti de la magie de l'exécution, débarrassé d'une écoute subjuguée et naïve fera la part des choses et n'associera qu'avec circonspection le chanteur et le canteur, ou plutôt dissociera le chanteur, professionnel de la mise 
en scène et de la mise en voix, de la personne réelle qu'il pourrait être dans la vie civile et que son physique aussi possiblement incarne (cf. July 2015) :

Quoi qu'il en dise, l'exécutant, fût-il l'auteur du texte, ne parle pas lui-même. L'emploi $\mathrm{du}$ je importe peu : la fonction spectaculaire de la performance ambiguïse assez ce pronom pour que se dilue dans la conscience de l'auditeur, sa valeur référentielle [...]. Pour l'auditeur, la voix de ce personnage qui s'adresse à lui n'appartient pas tout à fait à la bouche dont elle émane. (Zumthor 1983, 231)

L'opération du chant agit paradoxalement pour créer l'émotion mais pas forcément pour illusionner le spectateur/auditeur. Il faut à l'auditeur quelques suggestions supplémentaires, quelques indices de proximité entre les deux ethe (ethos intra-discursif du canteur et ethos extra-discursif du chanteur) pour s'orienter vers la piste autobiographique. Or c'est exactement l'attitude de Barbara qui s'affiche tout au long de son œuvre poétique et se met à l'avant-scène et qui, lorsqu'elle n'est pas le protagoniste de sa chanson, en est le témoin, privilégié et unique comme dans " Drouot ».

Cette manière toute particulière qu'a donc Barbara d'instaurer et de maintenir le dialogue avec le public tout en cryptant les souvenirs les plus douloureux, au point de rendre parfois compliqués ou hermétiques le message autobiographique, caractérise cette poétique de la seiche, qui se révèle en se masquant, voire qui se masque pour mieux s'autoriser à se révéler à ceux ou celles, qui dans le public, auraient, comme Marie Chaix, l'ouïe particulièrement fine ou sensible. Le je de Barbara n'est donc pas un locuteur quelconque et universel, une instance discursive artificielle ; il est celui par qui le dialogue s'instaure, ou mieux s'amorce, sans jamais tomber dans la confidence bavarde. Il est le héros de ces micro-aventures que chaque texte décrit. Par cette situation tout à fait exceptionnelle, la présomption, qui ne demande qu'à devenir certitude, qu'un espace autobiographique se creuse sous la chanson peut naître chez le lecteur/auditeur. Philippe Lejeune confirme ces nuances :

Dans la plupart des cas, le « je » des poèmes est un « je » sans référence, dans lequel chacun peut se glisser ; c'est le " prêt-à-porter » de l'émotion. La subjectivité universelle du lyrisme est assez différente du discours autobiographique, qui, lui, suppose une attitude de communication entre deux personnes distinctes et séparées. (Lejeune 1996, 245)

Il se pourrait fort que, grâce à cette " attitude de communication " qu'adopte la 'Dame brune ,, grâce à ce dialogue perpétuel, Barbara sorte de la "subjectivité universelle du lyrisme " pour atteindre un véritable « discours autobiographique ».

D'ailleurs le ressassement, perpétuel dans l'œuvre chantée de Barbara, donne aussi à l'auditeur l'illusion de la démarche autobiographique. Ainsi, on pourrait signaler les chansons qui, de loin en loin dans l'œuvre de Barbara, reprennent le schéma d'une liaison passionnelle entre une femme mûre et un jeune homme : du "Bel âge " (1964) à "Sables mouvants " (1993) en passant par " Amours incestueuses » (1972), «L'amour magicien » (1978) et « La 
déraison " (1981), nous retrouvons la même obsession, le même fantasme, mais surtout les mêmes images, les mêmes codes : l'utilisation des saisons pour décrire cette union parfois jugée hors nature, l'arrivée brutale, chaleureuse et ensoleillée du personnage masculin. Or ces reprises sont néanmoins évolutives, elles subissent le poids des années qui sont passées, de 1964 à 1993. Cette situation récurrente n’a pourtant jamais la même issue, comme si, de fait, les cinq chansons renvoyaient chacune à une expérience vécue, et vécue différemment selon l'âge des partenaires... et de la cantrice/chanteuse. Aucune ne ressemble tout à fait à l'autre : simple tentation refoulée dans "L'amour magicien ", elle est pleinement vécue dans "La déraison ». Le garçon s'en va dans "Le bel âge », c’est elle qui ‘ part au plus beau dans "Amours incestueuses ". L'automne des premières chansons devient l'hiver dans " La déraison » et au-delà dans "Sables mouvants » où le grand âge supprime toutes les craintes et tous les scrupules.

Mais si Barbara donne plus qu'un autre poète l'impression de s'être racontée, si nous pouvons la suivre dans cette entreprise de séduction autobiographique, c'est sans doute parce qu'en plus des chansons, elle a créé, à travers quelques interviews décisives et une centaine de textes un véritable espace autobiographique. Certaines affirmations sont assez catégoriques à ce sujet : "On radote toujours sur soi. Toujours se raconter soi. » (Perciot 2002, 42) Aux élèves du Cours Alice Dona qu'elle rencontre le 2 juillet 1993, elle explique : "Je n'ai aucune chanson dans mes tiroirs. Je ne sais même pas ce que c'est d'en avoir. Et en plus je n'ai aucune imagination. Alors, je n'écris que des choses qui sont bêtement ma vie et, j'espère, un peu celle de tout le monde. » (Dona 2007, 141) "Je n'écris que comme dans un journal intime ", confie encore Barbara aux journalistes (July 2012, 309). On le voit : même si ces explications ne sont pas à prendre au pied de la lettre, elles attestent l'existence d'un espace autobiographique, celui que dessinent les contours mouvants de la voix expulsant de la carapace d'une femme pudique des sons et des signes noirs, mots écrits, mots chantés, mots quoi qu'il en soit pénétrés d'une subtile substance subjective.

Pourtant, dans les chansons de Barbara, nous sommes bien loin de ce qu'on appellerait classiquement en littérature une œuvre autobiographique. Une mémoire volontairement fragmentaire, un cryptage savant du vécu, et surtout une identité particulièrement équivoque chez cette femme de l'ombre empêchent le lecteur spectateur de se livrer sans retenue à l'illusion autobiographique. À l'intrigante question "Barbara dit-elle la vérité ? ", on peut certes répondre par les pirouettes traditionnelles qui, du côté de la psychologie, font de l'introspection une chimère, et du côté de la littérature, font de tout écrit une fabrication : s'il est sans doute impossible d'atteindre la vérité, en particulier celle d'une vie humaine, les chansons et les textes déploient néanmoins suffisamment d'effets de vérité pour donner à l'amateur de Barbara le désir sinon d'atteindre une vérité définitive, du moins d'entreprendre la quête de quelques vérités. Et finalement, l'essentiel est là : est autobiographique ce qui donne l'impression aux lecteurs que la volonté de l'auteur est en effet de dévoiler son intimité. Lejeune propose à ce sujet une distinction fondatrice : "Un autobiographe n'est pas quelqu'un qui dit la vérité sur sa vie, mais quelqu'un qui dit qu'il la dit. » (Lejeune 1996, 42) 
Et Barbara ne nous trompe qu'à demi dans cette intention puisqu'elle prend la peine d'ajouter un possessif au mot c vérité > dès la chanson "Perlimpinpin " ("N'avoir que sa vérité ») et également dans «Amours incestueuses » ("Ceci est ma vérité / Du cœur de moimême ») : une vérité subjective n'est pas moins une vérité et constitue le régime humain le plus intéressant de la vérité. Cette vérité subjective se dépose tout particulièrement dans la représentation de l'espace et du temps, ces deux coordonnées fondamentales qui arriment tout sujet à son vécu.

\section{Jeu de cache-cache : les lieux de Barbara}

Dans cet espace autobiographique, voué à la clarté et à la transparence, une situation d'ambiguïté paradoxalement se met en place en raison de "l'incertitude où se trouve le lecteur de la position du narrateur par rapport à ce qu'il raconte " (Lejeune 1996, 166). Philippe Lejeune commente ainsi ce goût du camouflage, mené en funambule sur le fil de l'existence réelle. Se développant moins sous un pacte autobiographique que derrière un "pacte fantasmatique » (Lejeune 1996, 42), les chansons de Barbara comme les récits de Gide recréent les situations qui ont eu lieu en les transformant au risque de les dénaturer :

Si chaque ouvre contient sa part de confidence, ce serait naturellement une erreur complète que d'y voir des confessions [...]. Gide éprouve grande volupté à ces exercices qui lui permettent de dire « je » sur le mode de l'hypothèse, de la virtualité, sans tomber dans le « moi » autobiographique [...]. [I]l ne lui déplaît pas d'intriguer, de laisser lecteurs et critiques se tromper, quitte à les guider après coup... (Lejeune 1996, 168-169)

C'est pourquoi on peut parler à propos de Barbara d'autobiographie intermittente, d'autobiographie en pièces détachées ou encore d'autobiographie cryptée : Barbara crée des textes à clé, codés et décodables par elle seule (ou à la rigueur par ceux et celles qui la connaissent aussi bien qu'elle-même). Rien ne le montre mieux que les petits arrangements de Barbara avec les lieux familiers de Monique Serf. Ainsi, le personnage que Barbara semble inventer de toutes pièces dans " Hop-là " (1970) lui ressemble par plusieurs traits. Physiquement d'abord : elle le présente comme un être longiligne ; mais plus important encore, c'est l'état civil, et l'attestation de son lieu de naissance : « Rue d'Bagnolet, près de la rue des Pyrénées, / Quand je suis née $[\ldots]$ » (vers 3 et 4 ).

Vrai ou faux ? En réalité, Barbara est née le 9 juin 1930 à Paris, rue Brochant, près du square des Batignolles, dans le XVII ${ }^{e}$; mais elle vivra surtout, après la guerre, dans le $\mathrm{XX}^{\mathrm{e}}$ arrondissement de Paris, à l'Est, où se situe cette rue de Bagnolet. L'espace autobiographique, entendu ici au sens le plus concret, engage un pas de deux avec la vérité factuelle. Le square des Batignolles ne tardera pas à entrer dans la géographie de Barbara puisque, trois ans plus tard, la chanson "Perlimpinpin » le met en scène : "Le goût de l'eau, le goût du pain / Et celui du perlimpinpin / Dans le square des Batignolles. » 
Puis, en 1978, il réapparait comme le cadre de jeu des enfants de novembre, qui viennent de faire leur rentrée scolaire : " Il y a des rouges-gorges / Au jardin des Batignolles » (" Il automne", vers 35-36).

Parfois, l'œuvre poétique laisse apparaitre des ancrages topographiques plus explicites comme le jardin de Précy qui sera l'objet en 1978, d'une émouvante louange musicale, dans laquelle elle le compare à un square de l'enfance, justement. La chanson qui rend hommage à Esther Brodsky, Madame Serf, porte le nom du lieu qu'elle a hanté, Rémusat, puisque la mère de Barbara rejoignit sa fille jusqu'à sa mort dans un appartement confortable, rue de Rémusat, durant toutes les années 1960.

Ces petits arrangements avec l'espace invitent à se demander comment Barbara s'arrange avec le temps. Nous nous contenterons ici $^{8} \mathrm{~d}^{\prime}$ une seule indication générale, qui appellerait bien des spécifications. La plupart des chansons de Barbara sont entièrement tournées vers le souvenir. Nous insistons d'ailleurs sur le singulier car la chanteuse est souvent en quête de son passé et les souvenirs ne reviennent pas toujours si spontanément ou si abondamment. Il faut souvent une occasion favorable pour qu'ait lieu leur émergence. Ainsi dans "Mon enfance " (1968), c'est en revenant à Saint-Marcellin, au sud de l'Isère, que la mémoire est recouvrée : «Et j’ai retrouvé comme avant, / Longtemps après [...] / C’est fou, tout, j'ai tout retrouvé.»

Sur le modèle romantique, les lieux qui ont servi de cadre au passé permettent dans l'esprit du poète le défilé des événements qui y furent accueillis. Ainsi, dans la chanson "Nantes ", si le souvenir des lieux et des moments est bien inscrit dans la mémoire du poète, c'est tout de même le pèlerinage dans les rues de la ville bretonne qui va déclencher la verbalisation de ce souvenir. D'une certaine manière, guidée par sa tendance (ou son impuissance) à ne pas sortir de l'autobiographie, Barbara est toujours tentée en prenant la plume de fouiller sa mémoire.

\section{Conclusion}

Être de papier et personnage de scène, objet de fantasme pour son public, condamné à une exhibition programmée, plus qu'un autre artiste, le chanteur se tient dans un ambigu dédoublement. Si le pseudonyme de l'écrivain n'a pas forcément valeur d'altérité, ${ }^{9}$ Barbara, elle, est un personnage fabriqué :

Barbara chanteuse vit ses rêves sur scène. [...] De chanson en chanson, d'année en année, Monique Serf, la personne biographique, donne corps à une figure composite d'elle-même, à la fois poétique et spectaculaire, qu'elle nomme Barbara. Elle joue à s'y identifier, s'y identifie, s'identifie au je même de cette substitution qui suppose, pour bien prendre, que l'artiste coïncide pleinement avec l'image de soi ainsi projetée, tout en en gardant le contrôle et en en restant partiellement la spectatrice. (Blanckeman 2005, 2) 
Son existence complexe est donc plus difficile à restituer, fût-ce dans de micro-textes. Parfois Barbara emprunte quelques vestiges de Monique Serf mais c'est un je de projection qui investit la plus grande part des textes de l'artiste. Aussi lorsque Barbara se met au piano, il lui est bien difficile de certifier ce qu'elle raconte et d'authentifier les événements. C'est pourquoi elle a recours à un simple je lyrique, nécessaire puisque Barbara c'est elle, mais biographiquement inconsistant puisque Barbara n'existe pas réellement, n'existe que sur scène, n'existe que par le désir et l'imagination des spectateurs.

$\mathrm{Si}$, comme nous l'avons montré, l'œuvre de Barbara n'est pas parfaitement transparente, la cantrice n'en laisse pas moins partout l'empreinte de son propre passé, ou tout au moins, partout l'empreinte de ce que nous nous représentons comme son possible vécu. Elle accomplit un travail minutieux d'encodage : travail qu'elle remet très souvent sur le métier à tisser, comme pour brouiller davantage les pistes et rendre ses poèmes toujours plus mystérieux. Alors l'espace autobiographique atteint sa véritable densité : Monique Serf fait des aveux posthumes dans Il était un piano noir... qu'elle signe Barbara et laisse le soin à l'œuvre antérieure d'en dire plus. Barbara emprunte, brouille ou falsifie les souvenirs réels de Monique Serf ; Monique Serf distille ses émotions pour que Barbara les incarne à travers une image concrète, mais équivoque et mystérieuse. Il n'y a sans doute qu'un seul piano noir qui règne, souverain, dans l'imaginaire de la chanteuse ; mais sur le clavier, ce qu'on peut entendre, pour peu qu'on tende l'oreille, c'est bien un jeu à quatre mains qui se croisent et se répondent. (July 2014, 13)

\section{Notes}

1 D'autres universitaires reprennent à Lejeune cette théorie comme Julie Valéro à propos de l'œuvre théâtrale de Jean-Luc Lagarce par confrontation à son journal. Cf. Valéro 2012.

2 De fait, Barbara mourra sept mois plus tard, le 24 novembre 1997, d'un choc infectieux foudroyant.

3 À partir de 1964 et notamment du voyage à Göttingen, le récit jusque-là linéaire bien que très elliptique s'interrompt.

4 Dédicace explicite mais tardive. Pour une analyse des stratégies de mise en suspens dans la chanson « Nantes ", cf. July 2004, 35-41.

5 Marie Chaix fut étonnée, après la publication, en 1986, de l'ouvrage Barbara aux éditions Calmann-Lévy, que la chanteuse mît spontanément une certaine distance dans leur relation. Aujourd'hui, elle comprend à rebours qu'elle avait soulevé un lièvre en remarquant la parenté des trois chansons du triptyque et en dévoilant, trop tôt pour Barbara, Jacques Serf sous le plumage de « L'aigle noir».

6 Visible dans l'édition 2001 de N'avoir que sa vérité d'Alain Wodrascka.

7 Titre de l'album 1973 et d'une chanson de cet album qu'écrit pour elle François Wertheimer. 
8 Pour un long développement sur l'ambiguïté et le souci mémoriels chez Barbara et autour des questions de re-création et de mythographie, cf. July 2004, 211-231.

9 Lejeune 1996, 24 : «Les pseudonymes littéraires ne sont en général ni des mystères, ni des mystifications; le second nom est aussi authentique que le premier, il signale simplement cette seconde naissance qu'est l'écriture publiée. "

\section{Bibliographie}

Barbara : Il était un piano noir... (Mémoires interrompus). Paris : Fayard, 1998.

Blanckeman, Bruno : «Et Barbara nous parle». In : La lettre des amis de Barbara 22 (été 2005), 2-4.

Chaix, Marie : Barbara. Paris : Calmann-Lévy, 1986.

Cyrulnik, Boris : Les vilains petits canards. Paris : Odile Jacob, 2001.

Dona, Alice: Chanteuses ou coiffeuses. Paris : Éditions Anne Carrière, 2007.

Garcin, Jérôme : Barbara. Claire de nuit. Paris : La Martinière, 1999.

July, Joël : Les mots de Barbara. Aix-en-Provence : PUP, 2004.

July, Joël (éd.) : Barbara. L'intégrale. Paris : L'Archipel, 2012.

July, Joël : "N'y aurait-il pas plusieurs Barbara? »In : Sir, Libor / Laffeychine, François / Landete,

Pierre (éds) : Barbara. Album de photographie. Bordeaux : Castor Astral, 2014.

July, Joël : "Que reste-t-il de nos ethe ? ». In : Jousset, Philippe (éd.) : L’homme dans le style et réciproquement. Aix-en-Provence : PUP, 2015, 173-192.

July, Joël (éd.) : Chanson : du collectifà l’intime. Aix-en-Provence : PUP, 2016.

Lehoux, Valérie : Portrait en clair-obscur. Paris : Fayard/Chorus, 2007.

Leiris, Michel : L'âge d'homme. De la littérature considérée comme une tauromachie. Paris : Gallimard, 1998.

Lejeune, Philippe : Le pacte autobiographique. Édition augmentée. Paris : Points Seuil, 1996 [Paris : Le Seuil, 1975].

Lejeune, Philippe : L'autobiographie. Paris : CNED- L'Ecole des Lettres, 2002.

Perciot, Jacques : Barbara...sur parole. Paris : Éd. Didier Carpentier, 2002.

Tournier, Jacques : Barbara ou les parenthèses. Paris : Seghers, 1968.

Valéro, Julie : «Lagarce ou les heureux ratés d'un espace autobiographique ». In : Douzou, Catherine (éd.) : Lectures de Lagarce. Rennes: PUR, 2012, 63-78.

Wodrascka, Alain : N'avoir que sa vérité. Paris : Éditions Didier Carpentier, 2001.

Zumthor, Paul : Introduction à la poésie orale. Paris : Le Seuil, 1983.

\section{Discographie}

Barbara : Barbara chante ses plus grands succès de 1964 à 1974. Philips 9101-109, 1977 (33 tours). 